DOI: http://dx.doi.org/10.18310/2446-4813.2015v1n3p37-48

\title{
CONHECIMENTO SOBRE INFLUENZA ENTRE PROFISSIONAIS DE SAÚDE DE UM HOSPITAL GERAL
}

\author{
KNOWLEDGE ABOUT THE INFLUENZA AMONG HEALTH \\ PROFESSIONALS OF A GENERAL HOSPITAL
}

\section{Larissa Maria Isaac Maximo}

Médica Residente de Pediatria do Instituto Fernandes Figueira.

Email: larissamaximo@hotmail.com

\section{Nathalia Marcy Barbosa da Cunha}

Médica Residente de Pediatria do Instituto Fernandes Figueira / FIOCRUZ.

Email: nathaliamarcy@yahoo.com. br

\section{Maria Eduarda Pereira de Queiroz}

Médica Residente de Clínica Médica do Hospital Municipal Miguel Couto.

Email: eduarda_queiroz@hotmail. com

\section{Nataly Damasceno de Figueiredo}

Nutricionista, Sanitarista, Mestre em Ciências, Doutoranda em Saúde Pública.

Email: natalydamasceno@hotmail. com

\section{Resumo}

Em 2009 ocorreu uma pandemia do vírus Influenza A H1N1. Por conta da capacidade de disseminação do vírus, o mesmo foi alvo de preocupação por toda a sociedade. Com isso, observou-se a importância de profissionais de saúde capacitados para realização de diagnóstico e tratamento oportunos bem como medidas de prevenção e controle da epidemia. O objetivo é avaliar o conhecimento de profissionais da saúde sobre a Influenza H1N1. A pesquisa teve como público-alvo profissionais da saúde de um hospital geral do município do Rio de Janeiro, referência durante a epidemia de 2009. Aplicou-se um questionário durante a campanha de vacinação destinada a este grupo, realizada pelo Núcleo de Epidemiologia do hospital. De 1710 vacinados, 462 participaram da pesquisa. A média de idade foi de 38,9 anos (DP $\pm 11,96$ ), sendo $61,5 \%$ do sexo feminino e $33,3 \%$ do sexo masculino. Em relação à categoria funcional, $25,5 \%$ eram médicos, $39,6 \%$ enfermeiros e $34,6 \%$ da categoria "outros". $\mathrm{Na}$ autoavaliação do conhecimento sobre a doença, $45 \%$ classificaram seus conhecimentos sobre a doença como Muito Bom ou Bom. As pontuações médias obtidas dos conhecimentos sobre fatores de risco mostram um bom desempenho dos médicos, com média de 6,17 ( $\pm 2,9)$ acertos, quando o máximo seriam 10 . Os resultados apontam que o conhecimento demonstrado pelos profissionais de 
saúde é insuficiente. Este grupo tem papel fundamental de enfrentar provável segunda onda causada por este vírus. Assim, são importantes ações contínuas de educação e informação direcionadas a este público, bem como subsídio ao seu planejamento e organização.

Palavras-chave: Influenza; H1N1;

Profissionais de Saúde; Conhecimento.

\section{Abstract}

In 2009 there was a pandemic of the virus Influenza A H1N1. Because of its spreading capacity, it has been causing concern throughout society. Along the same, there was the importance of health professionals trained to perform diagnosis and timely treatment as well as prevention and control of the epidemic. The study was made with health professionals, who work in a general hospital, located in Rio de Janeiro, reference during the 2009 epidemic. During the vaccination campaign, we applied a questionnaire aimed at this group, conducted by the Hospital's Epidemiology Center. Of 1710 vaccinated, 462 participated in the study. The mean age was 38.9 years (SD \pm 11.96 ), and $61.5 \%$ female and $33.3 \%$ male. Regarding the functional category, $25.5 \%$ were doctors, $39.6 \%$ nurses and $34.6 \%$ category "other". In the self-assessment of knowledge about the disease, $45 \%$ rated their knowledge as Very Good or Good. The average score of knowledge about risk factors show a good performance of physicians, averaging 6.17 $( \pm 2.9)$ correct answers, when the maximum is 10 . The results show that knowledge demonstrated by health professionals is insufficient. This group has a fundamental role to face a prossible second wave caused by this virus. Thus, it's necessary to reinforce the importance of continuous education actions and information targeted to that audience, as well as the supporting of its planning and organization.

Keywords: Influenza; H1N1; Health Personnel; Knowledge.

\section{Introdução}

Ainfluenza ougripeéuma infecçãoviral aguda do sistema respiratório, de elevada transmissibilidade e distribuição global com uma freqüência sazonal, podendo se apresentar de forma mais ou menos grave. A doença se constitui em um importante problema de saúde pública, uma vez que pode evoluir com manifestações clínicas de maior gravidade e ainda por apresentar um elevado potencial pandêmico. Os vírus da influenza A e B possuem subtipos que sofrem contínuas mutações, dando origem a novas cepas. Estas apresentam, em geral, diferentes graus de distinção em relação àquelas até então circulantes facilitando, assim, a disseminação da doença diante da suscetibilidade da população e elevando o risco de epidemias ou pandemias. ${ }^{1: 495}$

Durante o século $X X$ ocorreram três pandemias provocadas por diferentes tipos de vírus Influenza, como a gripe espanhola (1918-1919), causada pelo vírus Influenza H1N1, em que as mortes foram estimadas de cinqüenta a cem milhões; a gripe Asiática (1957-1958), causada pelo vírus Influenza H2N2, e a gripe de Hong Kong (1968-1969), causada pelo vírus H3N2. Nas duas últimas, foram estimadas entre um a quatro milhões de mortes. ${ }^{2,3}$

A primeira pandemia do século XXI ocorreu no ano de 2009, decorrente da circulação entre seres humanos de um novo 
subtipo do vírus, Influenza A H1N1 2009, ficando a doença popularmente conhecida por "gripe suína". ${ }^{4}$ A genética deste tipo de Influenza provém da recombinação gênica ocorrida entre o vírus suíno, aviário e humano. Os primeiros casos foram confirmados, no sul da Califórnia. No mesmo período, no México, iniciou-se um surto de Doença Respiratória Aguda Grave, em conjunção com este novo vírus que, por sua vez, se disseminou pelo mundo rapidamente. ${ }^{3,5}$ Posteriormente foram diagnosticados casos nos Estados Unidos, Europa e até mesmo no Brasil. Neste, os primeiros foram confirmados em maio de 2009, sendo considerados casos alóctones, no entanto, logo foram identificados casos autóctones no Rio de Janeiro passando - Brasil a ser considerado um país com transmissão sustentada. ${ }^{6}$

Em todo o mundo, até 31 de Janeiro de 2010, mais de 209 países e territórios relataram casos da doença. Além disso, pelo menos 15.174 mortes foram atribuídas à doença. ${ }^{7}$

A Organização Mundial de Saúde (OMS) diante do risco de novas ocorrências de Influenza pandêmica mantém atualizado um Plano de Contingencia. Com base neste plano, no dia 11 de junho de 2009, elevou o nível de alerta pandêmico mundial para a fase seis (última fase). Esta ação foi motivada devido à rápida propagação do vírus e não pela gravidade da doença. ${ }^{3,5}$

A velocidade de disseminação do vírus e o aumento do número de casos impediram a confirmação laboratorial de todos, uma vez que a contagem do número de doentes não era considerado mais importante para o monitoramento do risco e para orientar a implementação de medidas de respostas. ${ }^{2}$ Por esse motivo, foram considerados casos confirmados de Influenza A H1N1 2009 aqueles com diagnóstico laboratorial através de sorologia e casos suspeitos, aqueles que tiveram contato próximo com um caso confirmado ou, ainda, pertencentes à mesma cadeia de transmissão (clínico-epidemiológica). ${ }^{5}$

No Brasil, o Ministério da Saúde estabeleceu as ações de controle a partir do protocolo da OMS. Através deste, foram implementados o fluxo de informações, a rede para investigação laboratorial, bem como fluxo de pacientes de acordo com a classificação de risco. As informações sobre a doença, como forma e período de transmissão, sintomas gerais e os que sinalizavam maior risco foram amplamente divulgados pela mídia para orientar a população. Entre os profissionais de saúde a divulgação também se deu através de informes técnicos e protocolos de manejo clínico. ${ }^{8}$

A transmissão da Influenza A H1N1 2009 e suas manifestações clínicas mostraram-se semelhantes à da influenza sazonal. A maioria dos indivíduos que se infectaram evoluiu para a cura, e as complicações e mortalidade mostraramse também semelhantes ao verificado na ocorrência sazonal da doença. ${ }^{5}$ Entretanto, apesar da semelhança na dinâmica de transmissão, o aumento do número de casos desse novo subtipo destacou a necessidade de revisão das medidas de prevenção e controle a todos os profissionais de saúde que prestam assistência direta ao paciente, como também aos familiares e contactantes. $^{9}$

Considerou-se caso suspeito de Influenza A H1N1 2009 todos os indivíduos com doença respiratória aguda caracterizada por febre superior a $38^{\circ} \mathrm{C}$, tosse, dispnéia, acompanhadas ou não de dor de garganta e manifestações gastrointestinais. ${ }^{5}$ Porém, além do diagnóstico clínico, deveriam ser avaliados os fatores de risco, que podem contribuir para o agravamento do quadro gripal. $^{9}$

Embora tenha ocorrido em época distinta às anteriores, esta pandemia apresentou muitos aspectos semelhantes a outras de origem respiratória, dentre 
eles um dos que mais podemos ressaltar é o relacionado aos grupos de risco. ${ }^{10}$ Os principais foram gestantes, menores de dois anos, pacientes com mais de 60 anos, imunodeprimidos, portadores de doença cardiovascular, hematológica, hepática, metabólica, neurológica e renal., 3

Uma das principais estratégias de controle e contenção de epidemias de doenças com transmissão por via respiratória está na veiculação de informações entre a população e profissionais de saúde através da mídia e informes técnicos. Nesta epidemia, foram disseminadas amplamente informações gerais sobre medidas de proteção individual, modos de transmissão da doença e grupos e fatores de risco que poderiam levar a casos graves bem como aumentar a suscetibilidade dos indivíduos. ${ }^{9}$

Diante do exposto, é importante que haja a disseminação do conhecimento entre profissionais de saúde, visando à identificação de grupos de risco, bem como a adoção de medidas de proteção individuais, possibilitando o controle destas e de outras doenças infecciosas e a redução de sua letalidade. Estas ações são relevantes não só no enfrentamento da influenza como também no que diz respeito a outras epidemias por doenças transmissíveis, sejam elas emergentes ou reemergentes.

Este trabalho teve como objetivo comparar o conhecimento dos profissionais de saúde com o de profissionais de áreas que não da saúde que trabalham em um hospital geral, localizado no Município do Rio de Janeiro, sobre comportamento, formas de transmissão e fatores de risco da Influenza A H1N1 2009.

\section{Material e métodos}

Foi realizado um estudo transversal que teve como público alvo profissionais da saúde lotados em um hospital geral, localizado no município do Rio de Janeiro, que atuou como referência durante a pandemia de 2009.

Para coleta de dados, foi utilizado um questionário de autoresposta, aplicado durante a campanha de vacinação realizada no hospital, que contava com um quadro de 2.900 funcionários, em março de 2010.

Este estudo analisou informações sobre os profissionais médicos ( $n=119)$, corpo de enfermagem $(n=183)$ e, para estabelecer comparações, utilizou-se os profissionais sem formação na área da saúde $(n=160)$, alcançando uma população total de 462 indivíduos. O grupo de comparação será tratado como "outros".

Os profissionais que aceitaram participar da pesquisa, o fizeram após ciência e assinatura do termo de consentimento livre e esclarecido. $O$ estudo foi aprovado por um comitê de ética em pesquisa da Escola de Medicina da Fundação Técnico Educacional Souza Marques.

O questionário contemplou informações demográficas (sexo e idade), categoria funcional, autopercepção dos conhecimentos sobre a doença, período de transmissibilidade, formas de transmissão, sintomas e grupos de risco para casos graves da doença.

Apesar das limitações da utilização de questionário de autoresposta, optouse por este tipo de coleta para que se pudesse alcançar um maior número de profissionais. Durante o período de coleta de dados, sempre esteve presente um dos pesquisadores, que passaram informações gerais sobre 0 preenchimento dos questionários sem interferir nas respostas.

O projeto de pesquisa foi aprovado pelo Comitê de Ética em Pesquisa da Escola de Medicina Souza Marques da Fundação Técnico Educacional Souza Marques, sob o parecer $n \div$. 001/2010. 


\section{Análise dos dados}

Foi feita uma analise descritiva das variáveis do estudo. Para medir os conhecimentos dos profissionais sobre transmissão, foi utilizada uma pontuação média que poderia variar de 0 a 10, atribuindo-se 1 ponto para cada acerto. Para avaliar os conhecimentos sobre fatores de risco e conhecimentos gerais, a pontuação média poderia variar de 0 a 5 .

Por não haver parâmetro para comparação dos níveis de conhecimentos dos profissionais médicos e do corpo de enfermagem, optou-se por estabelecer comparações com o grupo "outro". Comparando a Proporção de acerto através da Razão de Proporções (RP), utilizando-se o Teste Qui quadrado e intervalo de confiança de $95 \%$ para avaliar a significância estatística dos resultados.

Os dados foram armazenados e analisados através do programa Epi Info versão 3.5.1.

\section{Resultados}

Na população estudada, a média de idade foi de 38,9 anos ( $D P \pm 12,05$ ), sendo $61,5 \%$ do sexo feminino, $33,3 \%$ do sexo masculino e 5,2\% ignorado. Em relação à categoria funcional observou-se que $25,5 \%$ eram médicos, $39,6 \%$ do corpo de enfermagem e $34,6 \%$ do grupo "outros".

Autopercepção dos níveis de conhecimento sobre a doença

Aproximadamente $\quad 45 \%$ dos profissionais que participaram da pesquisam classificam seus conhecimentos sobre a doença como Muito Bom ou Bom. Os médicos tendem a apresentar uma classificação melhor quando comparados ao corpo de enfermagem e aos outros profissionais, que apresentaram perfil muito semelhante (Figura 1).

\section{Figura 1}

\section{Autopercepção dos níveis de conhecimento}

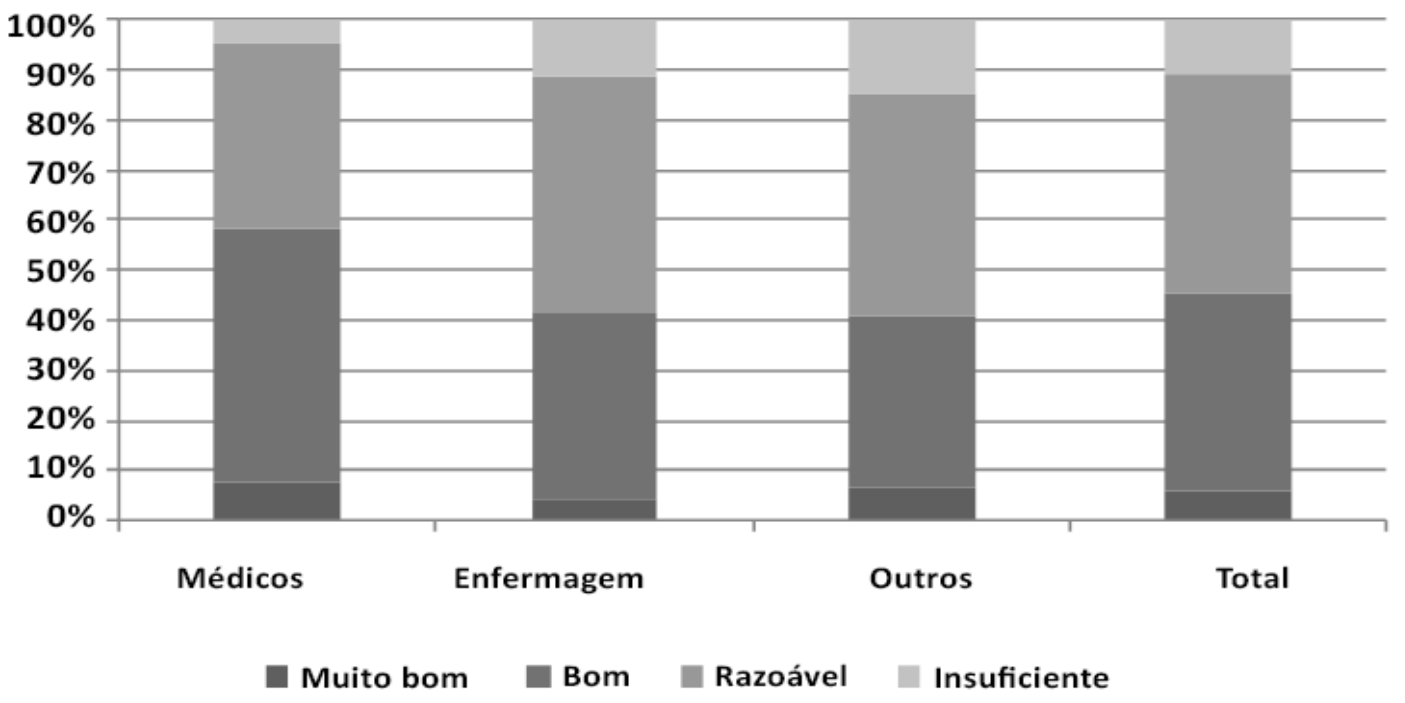

Fonte: Dados da pesquisa. 
Identificação de Grupos e fatores de risco

As pontuações médias obtidas a partir dos conhecimentos sobre fatores de risco mostram um bom desempenho dos médicos que atingiram uma Média de $6,17( \pm 2,9)$ quando o máximo seria 10 (Tabela 1). Tendo a categoria profissional "outros" como referência, verificase que os médicos apresentam conhecimento 3,03 vezes maior $(p<0,5)$ enquanto para os profissionais da enfermagem a RP foi $1,63(p<0,05)$.

Tabela 1

\begin{tabular}{c|cccc}
\hline \multicolumn{6}{c}{ Comparação das pontuações médias sobre grupos e fatores de risco } \\
\hline $\begin{array}{l}\text { Categoria } \\
\text { Funcional }\end{array}$ & Média & \pm DP & Diferença das Médias (IC 95\%) & p valor \\
\hline Médicos & 6,17 & 2,9 & $3,03(2,43-3,62)$ & 0,0000001 \\
Enfermagem & 4,60 & 2,9 & $1,63(1,07-2,19)$ & 0,0000001 \\
Outros & 2,97 & 2,2 & - & - \\
\hline
\end{tabular}

Fonte: Dados da pesquisa.

Os resultados sobre a identificação dos fatores de risco para complicações, apontam para um maior esclarecimento em relação ao grupo de gestantes, seguido por crianças menores de 2 anos e por fim os idosos. Os médicos mantiveram o percentual de acertos acima de $60 \%$ nas três opções analisadas, entre os profissionais da enfermagem observa-se um menor nível de conhecimento sobre os fatores de risco relacionados à idade, apresentando a proporção de acertos abaixo de $60 \%$ (Tabela 2).

Em relação aos grupos de risco, representados por agravos e doenças que aumentam a probabilidade de complicações, observa-se uma maior indicação de imunodeprimidos pelas três categorias analisadas. Entre os médicos foram mais identificadas, além da imunossupressão, as doenças metabólicas $(68,1 \%)$ e doença renal $(67,2 \%)$.

Tabela 2

(continua)

\begin{tabular}{c|ccc}
\hline \multicolumn{4}{c}{ Conhecimento sobre fatores de risco segundo categoria funcional } \\
\hline Grupo de Risco & Médicos & $\begin{array}{c}\text { \% de acertos (IC 95\%) } \\
\text { Enfermagem }\end{array}$ & Outros \\
\hline $\begin{array}{c}\text { Gestante } \\
\text { Crianças menores de } 2 \\
\text { anos }\end{array}$ & $89,9(83,0-94,7)$ & $85,2(79,3-90,0)$ & $73,1(65,6-79,8)$ \\
$\begin{array}{c}\text { Pessoas com mais de } 60 \\
\text { anos }\end{array}$ & $69,7(60,7-77,8)$ & $57,9(50,4-65,2)$ & $41,3(33,5-49,3)$ \\
& $61,3(52,0-70,1)$ & $49,7(42,7-57,2)$ & $38,1(30,6-46,1)$
\end{tabular}


(conclusão)

Imunodeprimidos

Doenças metabólicas

Doença Renal

Doença Hematológica

Doença Cardíaca

Doença Hepática

Doença Neurológica

$\begin{array}{lll}89,1(82,0-94,1) & 77,6(70,9-83,4) & 48,1(40,2-56,2) \\ 68,1(58,9-76,3) & 33,9(27,1-41,2) & 22,5(16,3-29,8) \\ 67,2(58,0-75,6) & 39,3(32,3-46,8) & 21,3(15,2-28,4) \\ 48,7(39,5-58,1) & 38,3(31,2-45,7) & 19,4(13,6-26,4) \\ 48,7(39,5-58,1) & 24,6(18,5-31,5) & 11,9(7,3-17,9) \\ 39,5(30,7-48,9) & 27,3(21,0-41,2) & 12,5(7,8-18,6) \\ 31,9(23,7-41,1) & 20,2(14,7-26,8) & 6,3(3,0-11,2)\end{array}$

$2(14,7-26,8)$

$6,3(3,0-11,2)$

Fonte: Dados da pesquisa.

A opção "Imunodeprimidos" apresentou a maior proporção de acertos quando comparado aos outros fatores de risco. Observou-se um percentual de $89,1 \%$ de acertos na categoria Médicos, $77,6 \%$ na Enfermagem e 48,1\% nos Outros.

Em "Doenças metabólicas" constatou-se um percentual menor de acertos quando comparado ao anterior, no qual a categoria Médicos apresentou 68,1\%, Enfermagem 33,9\% e Outros 22,5\%. O fator de risco "Doença renal" apresentou 67,2\% de acertos na categoria Médicos, 39,3\% na Enfermagem e 21,3\% nos Outros, enquanto "Doença hematológica" apresentou um percentual ainda menor, de 48,7\% nos Médicos, 38,3\% na Enfermagem e $19,4 \%$ nos Outros. Já em "Doença cardiovascular" o percentual foi de $48,7 \%$ nos Médicos, $24,6 \%$ na Enfermagem e 11,9\% nos Outros.

No grupo "Doenças hepáticas", observou-se um percentual ainda menor em todas as categorias, de 39,5\% nos Médicos, 27,3\% na Enfermagem e 12,5\% nos Outros.

$\mathrm{O}$ fator de risco que apresentou um menor percentual de acerto em todas as categorias foi "Doença neurológica", no qual somente 31,9\% dos médicos, 20,2\% da Enfermagem e 6,3\% dos Outros, selecionaram-no como de risco.

\section{Conhecimentos sobre sintomatologia dos casos graves}

A avaliação sobre sintomas relacionados aos casos graves da doença e que deveriam ser encaminhados às unidades de referência, apontou que o corpo de enfermagem e outros profissionais identificam a febre como principal ( $80 \%$ e $85 \%$, respectivamente), entre os médicos o sintoma mais apontado foi dispnéia (96\%).

A comparação das médias obtidas pelas diferentes categorias funcionais apontou que a categoria Outros pontuou 0,55 a mais do que os profissionais médicos $(p<0,05)$. Comparandose à enfermagem, não houve diferença significativa $(p>0,05)$. 
Período de transmissibilidade

Esta análise permitiu verificar uma baixa proporção de acertos em relação ao período de transmissão da Influenza A H1N1 2009. Os Médicos obtiveram uma maior pontuação $(48,7 \%)$, seguidos pela Enfermagem (20,9\%) e pela categoria Outros $(19,0 \%)$, sendo que estes últimos apresentaram resultados semelhantes (Figura 2).

\section{Figura 2}

\section{Conhecimento sobre período de transmissão segundo categoria funcional}

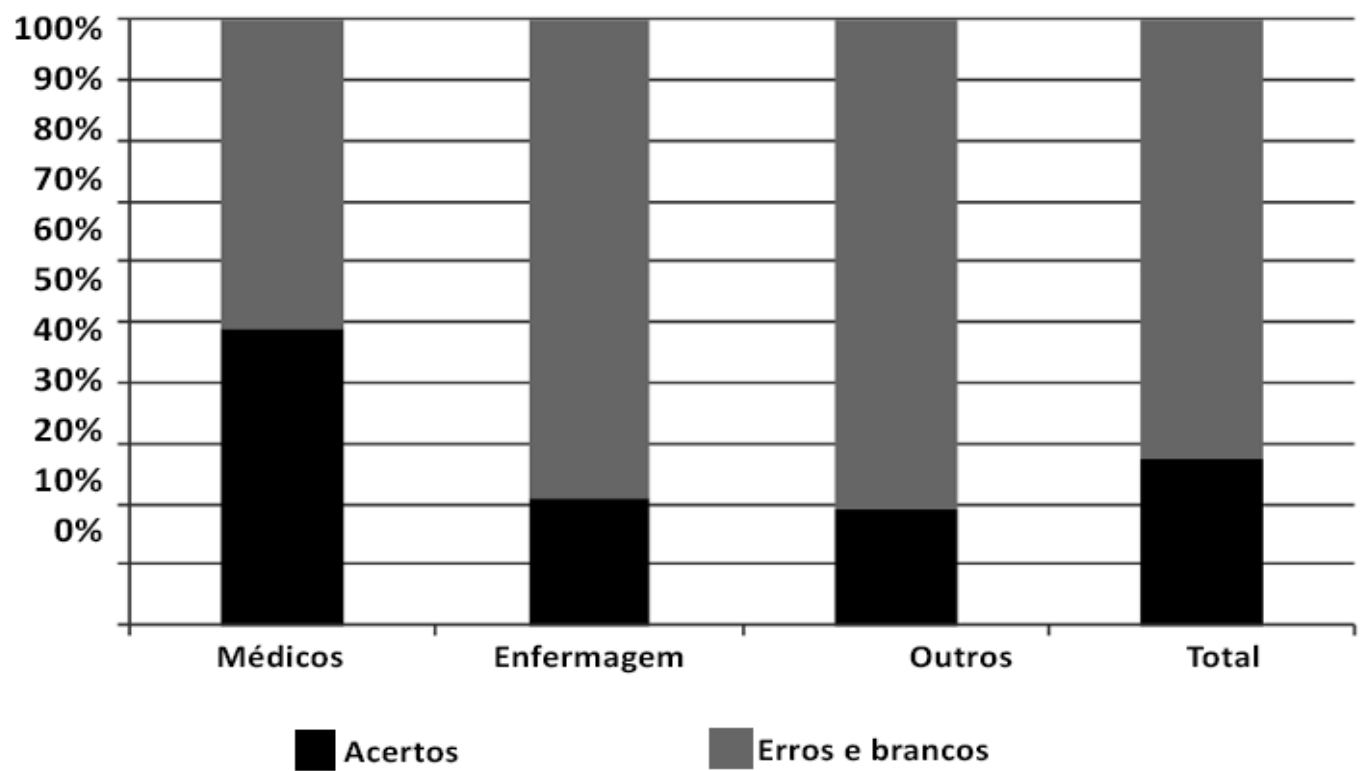

Fonte: Dados da pesquisa.

Em comparação aos profissionais que não são da área da saúde não houve diferença com a Enfermagem ( $R P=1,1$ (IC - 0,72 - 1,69) - $p$ valor $>0,05)$. Os Médicos apresentaram uma proporção de acertos 2,57 maior do que os profissionais de outras áreas ( $R P=2,57$ (IC 95\% $1,77-3,72)-p$ valor $=0,0000001)$.

Conhecimentos gerais sobre a doença

$\mathrm{Na}$ afirmativa sobre a transmissão sustentada, mostrava-se presente a definição da mesma, para evitar que o participante não assinalasse por não saber o significado. Ao avaliarmos os questionários observou-se que assim como as demais questões verdadeiras os médicos $(62,1 \%)$ foram os que tiveram melhor resultado, compatível com o nível de conhecimento autoreferido (Tabela 3 e Figura 1). 
Tabela 3

\begin{tabular}{c|ccc}
\hline \multicolumn{4}{c}{ Conhecimentos gerais segundo categoria funcional } \\
\hline Afirmativas & Médicos & Enfermagem & Outros \\
\hline $\begin{array}{c}\text { Lavar as mãos é uma } \\
\text { medida de proteção contra } \\
\text { a doença }\end{array}$ & $89,1(82,0-94,1)$ & $88,0(82,4-92,3)$ & $71,9(64,2-78,7)$ \\
$\begin{array}{c}\text { Existe transmissão } \\
\text { sustentada do vírus }\end{array}$ & $63,0(53,7-71,7)$ & $51,9(44,4-59,3)$ & $41,3(33,5-49,3)$ \\
$\begin{array}{c}\text { O diagnóstico pode ser } \\
\text { feito a partir de amostra } \\
\text { de secreções de nariz e } \\
\text { faringe }\end{array}$ & $66,4(57,2-74,8)$ & & \\
\hline
\end{tabular}

Fonte: Dados da pesquisa.

\section{Discussão}

Os resultados apontaram que os médicos classificam melhor seu nível de conhecimento sobre a doença ( $58 \%$ bom ou Muito bom). Apesar de serem profissionais de saúde, a autoclassificação dos conhecimentos do corpo de enfermagem foi muito próxima dos profissionais sem formação na área de saúde. Quando questionados sobre como classificariam seus níveis de conhecimento sobre a doença, $58 \%$ dos médicos entrevistados, $41 \%$ da categoria corpo de enfermagem e $40 \%$ da categoria outros profissionais, classificaram-se como um tendo um nível adequado (bom ou muito bom) de conhecimento.

Em pesquisa realizada em um Hospital do Peru, após a realização de um curso de capacitação com informações gerais sobre a Pandemia, observou-se um resultado semelhante aos apresentados no presente trabalho, onde o nível de adequação foi melhor entre os médicos se comparados aos enfermeiros. ${ }^{3}$

Vale ressaltar a importância do conhecimento relativo ao período de transmissão entre os médicos e o corpo de enfermagem, pelo fato destes estarem freqüentemente em contato com os pacientes e, portanto, necessitaram de um maior conhecimento para triagem, assistência e principalmente orientação aos pacientes.

De acordo com a literatura diversos aspectos dessa pandemia, ocorrida em 2009, se fizeram de forma semelhante a outras epidemias respiratórias. Mesmo tendo ocorrido em décadas diferentes e sendo provocadas por cepas distintas essas características se mantiveram. Principalmente no que diz respeito aos grupos mais suscetíveis e/ou que apresentam maior risco de desenvolver casos graves ${ }^{10}$. No entanto, o presente estudo apontou que os profissionais ligados diretamente ou indiretamente a área de saúde muitas vezes desconheciam os grupos de risco pertencentes a essa doença. Situação esta que ficou mais evidente na categoria 
enfermagem, que apresentou baixa proporção de acertos (abaixo de $40 \%$ ) na indicação dos grupos de risco.

Além da comparação entre as categorias profissionais, chama atenção, de maneira geral a não identificação de alguns grupos de risco, como doença hepática, neurológica e metabólicas.

A falta de conhecimento sobre os grupos de risco mostra-se como um problema alarmante, afinal somente conhecendo os indivíduos que fazem parte desse grupo seria possível realizar uma prevenção direcionada e mais efetiva, tanto no sentido de evitar a infecção, quanto no intuito de diminuir a incidência de casos graves e óbitos. Conseguir identificar a população mais vulnerável é fundamental para evitar e conter pandemias, além de reduzir a letalidade.

Levando-se em consideração que as características apresentadas nos diferentes tipos de influenza são semelhantes, destacase ainda que a não identificação destes grupos pode comprometer a assistência e prevenção de complicações nos casos de influenza sazonal, que chegam com frequência na rotina das emergências.

No que diz respeito ao desconhecimento dos profissionais sem formação na área de saúde, principalmente em relação aos grupos de risco representados pelas gestantes, menores de 2 anos e maiores de 60 anos, também nos faz refletir sobre o alcance dos métodos utilizados para transmitir essas informações para a população de maneira geral.

Apesar de não encontrar na literatura parâmetros para estabelecer uma comparação, considerou-se baixo a proporção de acertos dos enfermeiros e até mesmo dos médicos sobre o período de transmissibilidade da doença, visto que está é uma informação fundamental para identificação dos casos (história epidemiológica) e medidas de precaução (isolamento).
Em relação à lavagem das mãos como medida de prevenção observou-se um percentual de acertos elevados nos três grupos. Entretanto, apresentaram resultados aquém dos esperados, visto que esta é uma medida importante e constantemente divulgada à população, incluindo os profissionais de saúde, de modo que ainda deve ser enfatizada. A lavagem das mãos também é destacada como uma importante medida de prevenção pelo CDC (Centers for Disease Control and Prevention) e pela Association for Professionals in Infection Control and Epidemiology. ${ }^{11}$

Destaca-se que o uso do álcool não substitui a lavagem das mãos. Como uma maneira de lavagem das mãos, o uso do álcool (sob as formas em gel ou solução) é conveniente e auxilia a sua execução, porém somente quando estas não estiverem visivelmente sujas. Isso porque tal vírus é rapidamente inativado em trinta segundos após a antissepsia com álcool 70\%. ${ }^{6}$

Em relação à transmissão da doença, observou-se que a população do estudo mostrou-se com um conhecimento insuficiente. Devido ao grande potencial de transmissão de uma pessoa para a outra, por meio da inalação de gotículas infectadas de tosses ou espirros, ou por meio da contaminação das mãos ou superfícies, ${ }^{2}$ cabe ressaltar a importância dessa informação para que medidas de prevenção e controle da doença possam ser evitadas.

Além disso, o conhecimento relativo à forma de transmissão se mostra importante no manejo do paciente, de maneira a proteger o profissional de saúde e outros suscetíveis. No caso da Influenza é importante que haja o isolamento do paciente no ambiente hospitalar, com quarto privativo, vedação da porta do quarto e boa ventilação, sendo mantido até o descarte do diagnóstico de influenza ou enquanto ainda estiver durante o período de transmissibilidade da doença. Por essa razão, o contato dos profissionais de saúde 
com o paciente deve ocorrer com o uso de máscara N95, luvas, óculos, avental e gorro. Já o paciente deve utilizar máscara cirúrgica desde o momento de suspeita da infecção até a chagada ao local de isolamento. ${ }^{6}$

Do mesmo modo, os métodos diagnósticos também não demonstraram ser muito conhecidos pelos profissionais de saúde, apesar de haver uma maior proporção de acertos entre os médicos, com uma grande disparidade em relação à categoria "outros".

O diagnóstico da doença pode ser realizado por meio da técnica do aspirado de nasofaringe com frasco coletor de secreção. Alternativa é a técnica de aspirado de nasofaringe e orofaringe, exclusivamente com swab de rayon. O sangue e outras amostras são utilizados apenas para o monitoramento da evolução clínica do paciente e para a realização do diagnóstico diferencial. ${ }^{6}$

\section{Conclusão}

O estudo em questão buscou descrever o conhecimento dos profissionais de saúde em relação à Influenza A H1N1, permitindo uma avaliação da capacitação dos mesmos a respeito de conhecimentos mínimos necessários para ações de classificação de risco dos pacientes, orientações e ações de controle da pandemia.

De um modo geral, foi possível apontar deficiências no conhecimento sobre a doença apresentado pelos profissionais de saúde. Tal resultado revela a necessidade de atualizações e capacitações freqüentes, uma vez que esta é dinâmica, principalmente diante da constante eminência de pandemias.

Por essa razão, mostra-se importante o conhecimento pormenorizado sobre as doenças de um modo geral, principalmente em relação às emergentes e às reemergentes doenças estas que não fazem parte da rotina e necessitam de uma boa capacitação para diagnóstico, tratamento adequado e ações gerais de controle.

\section{Referências}

1. Brasil. Ministério da Saúde. Fundação Nacional de Saúde. Guia de Vigilância Epidemiológica. 6ed., Brasília; 2006. Volume II.

2. Kiely PM, Lian KY, Napper G, Lakkis C. Influenza A (H1N1) and infection control guidelines for optometrists. Clinical and Experimental Optometry, 2009 92: 490-494.

3. Bravo $\mathrm{K}$ et al. Nivel de conocimientos sobre la influenza A H1N1 en los trabajadores de salud del Hospital Nacional Arzobispo Loayza, Perú. Rev. peru. epidemiol. 13(2); Agosto 2009. Disponível em: <http://rpe.epiredperu.net/rpe_ediciones/2009_v13_n02/AO1_Vol13_ No2_2009_Conocimiento_influenza_A_H1N1_HNAL.pdf>. Acessado em: 15 ago 2011.

${ }^{4}$. Brasil. Ministério da Saúde. Secretaria de Atenção à Saúde. Departamento de Atenção Básica. Emergência de Saúde Pública de Importância Internacional - ESP II. Diretrizes para o Enfrentamento à Pandemia de influenza A (H1N1): Ações da Atenção Primária à Saúde. Brasília: Ministério da Saúde; 2009. Disponível em: <http://portal.saude.gov.br/portal/arquivos/pdf/ protocolo_influenzaa_aps_atualizado.pdf>. Acesso em: 15 mar 2010. 


\section{Artigo Original}

5. Secretaria de Estado da Saude de Sao Paulo. Divisão de Doenças de Transmissão Respiratória. Centro de Vigilância Epidemiológica "Prof. Alexandre Vranjac". Coordenadoria de Controle de Doenças. Características dos casos notificados de Influenza A/H1N1. Rev. Saúde Pública [Internet]; 2009 Oct [cited 2011 Feb 16] 43(5):900-904. Available from: http://www.scielosp. org/scielo.php?script=sci_arttext\&pid=S0034-89102009000500024\&lng=en. http://dx.doi. org/10.1590/S0034-89102009000500024.

${ }^{6}$. Machado AA. Infecção pelo vírus Influenza A (H1N1) de origem suína: como reconhecer, diagnosticar e prevenir. J. bras. pneumol. [Internet]; 2009 May [cited 2011 Aug 20] 35(5): 464-469. Available from: http://www.scielo.br/scielo.php?script=sci_arttext\&pid=S1806$37132009000500013 \&$ Ing=en. http://dx.doi.org/10.1590/S1806-37132009000500013.

7. Rachiotis G, Mouchtouri VA, Kremastinou J, Gourgoulianis K, Hadjichristodoulou C. Low Acceptance of vaccination against the 2009 pandemic influenza A (H1N1) among healthcare workers in Greece. Eurosurveillance, 15(6); 11 February 2010. Disponível em: <http://www. eurosurveillance.org/ViewArticle.aspx?Articleld=19486>. Acesso em 15 fev de 2010.

8. Brasil. Informe Epidemiológico Influenza Pandêmica (H1N1) 2009. Situação epidemiológica da Influenza Pandêmica (H1N1) 2009 no Mundo e no Brasil, até a Semana Epidemiológica 47 de 2009 Ano 1(11); Dezembro 2009. Disponível em: <http://portal.saude.gov.br/portal/ arquivos/pdf/boletim_influenza_se_47.pdf> Acesso em: 15 mar 2010.

9. Brasil. Emergência de Saúde Pública de Importância Internacional - ESPII. Protocolo De Manejo Clínico E Vigilância Epidemiológica Da Influenza - Versão III. Brasília, 5 de agosto de 2009. Disponível em: <http://portal.saude.gov.br/portal/arquivos/pdf/protocolo_de_ manejo_clinico_05_08_2009.pdf>. Acesso em: 15 mar 2010.

${ }^{10}$. Junior APN et al. Apresentação clínica e evolução de pacientes com infecção por Influenza A H1N1 que necessitaram de terapia intensiva durante a pandemia de 2009, Hospital São Camilo, São Paulo, 2010.

${ }^{11}$. White C, Kolble R, Carlson R, Lipson N (2005) The impact of a health campaign on hand hygiene and upper respiratory illness among college students living in residence halls. Journal of American College Health 53: 175-181. 